Z. Klin. Chem. Klin. Biochem.

12. Jg. 1974 , S. $464-467$

\title{
Quantitative Bestimmung dünnschichtchromatographisch getrennter Lipide aus Serum und Gewebe durch UV-Absorptionsmessung in Remission
}

Von U. Murawski, H. Egge und F. Zilliken

Physiologisch Chemisches Institut der Universität Bonn

(Eingegangen am 1.1. Januar/21. Juni 1974)

Die UV-Absorptionsmessung in Remission im Bereich von 196-210 nm erlaubt den direkten und quantitativen Nachweis dünnschichtchromatographisch getrennter Lipide. Sie hat gegenüber den densitometrischen Methoden nach Veraschung den Vorteil eines zerstörungsfreien Nachweises, der weitere anschließende Analysen erlaubt. Die Nachweisbarkeit im angegebenen Wellenlängenbereich ist an das Vorhandensein mindestens einer Doppelbindung im Molekül geknüpft. Die Nachweisgrenze liegt bei etwa 0,1 $\mu \mathrm{g}$.

\section{Quantitative determination of serum and tissue lipids by UV-absorption in remission after thin layer chromatography}

Lipids separated by thin layer chromatography can be detected and quantitatively determined by measuring UV-absorption in remission in the range of $196-210 \mathrm{~nm}$. Compared with densitometric evaluations after charring this method allows the additional subsequent use of other analytical procedures due to a destruction-free determination. Lipids containing at least one double bond can be detected down to $0.1 \mu \mathrm{g}$ in the given wavelength range.

Die quantitative Bestimmung der Lipide des Serums oder von Gewebsproben nimmt in der biochemischen und der klinisch-chemischen Analytik einen breiten Raum ein, wobei der Dünnschichtchromatographie eine zentrale Stellung zukommt (1). Jedoch bereitet die quantitative Auswertung der Dünnschichtchromatogramme, sei es densitometrisch nach Veraschen oder durch andere spezifische Nachweismethoden, experimentelle und apparative Probleme, die diese Bestimmung nicht ohne weiteres für eine Routinemethode geeignet erscheinen lassen (2). Einige dieser Schwierigkeiten können durch die UV-Absorptionsmessung in Remission (0/45 - Einfallwinkel/Meßwinkel) bei $196 \mathrm{~nm}$ umgangen werden. Gegenüber anderen Nachweismethoden, die entweder nach Veraschen oder Hydrolyse an den Nachweis spezifischer Bausteine geknüpft sind, erlaubt die UV-Absorptionsmessung anschließende zusätzliche analytische Schritte, wie z. B. die gas-chromatographische-, IR-, NMR-1) oder massenspektrometrische Bestimmung bestimmter Bausteine.

\section{Methodik}

Alle Lösungsmittel zur Extraktion, Verteilung und Chromatographie wurden redestilliert oder waren Uvasol-Qualität. Sojalecithin (Nattermann, Köln) enthielt $\leqslant 0,5 \%$ Lysoverbindung.

\footnotetext{
1) Nicht übliche Abkürzungen:

CHE Cholesterinester NMR Kernresonanz

TG Triglyceride DC \} Dünnschicht-

CH Cholesterin TLC chromatographie

FFS freie Fettsäuren UV Ultraviolett

PL Phospholipide GC Gas-Chromatographie

IR Infrarot
}

Bei allen anderen verwendeten Lipiden konnten dünnschichtchromatographisch keine Verunreinigungen nachgewieșen werden. Glasplatten $100 \times 100 \mathrm{~mm}$ wurden durch Tauchen in eine Suspension von $30 \mathrm{~g}$ Kieselgel (Camag DO) in $100 \mathrm{ml}$ Chloroform beschichtet. Vorbereitung der Platten und der Gelsuspension erfolgten in der angegebenen Weise (3). Die quantitative Applikation von Lipidproben erfolgte volumetrisch mittels einer AglaSpritze (Desaga) oder einer Konstriktionspipette ( $5 \mu$ l, Beckman). Die Meßanordnung mit dem Zeiss-ChromatogrammSpektrophotometer ist in Abbildung 1 schematisch wiedergegeben. Die jeweiligen Meßflächen wurden den chromatographischen Fleckengrößen angepaßt bzw. auf maximalen Ausschlag optimiert. Die Registrierung erfolgte über einen Servogor-SSchreiber mit Integrator.

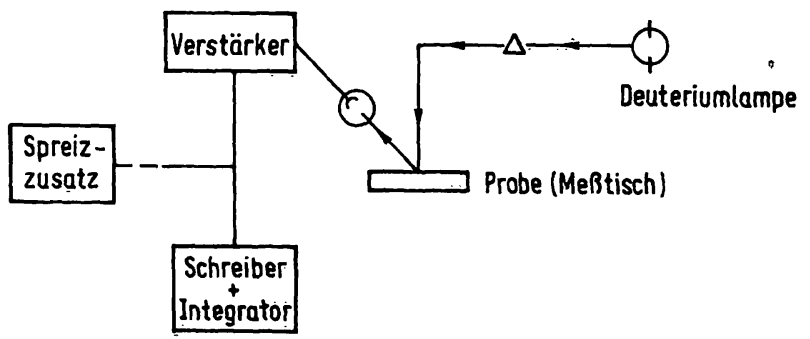

Abb. 1. Schema der Meßanordnung zur UV-Absorptionsmessung in Remission 0/45.

Zur Bestimmung des Einflusses von Zahl und Lage von Doppelbindungen auf die Flächenintegrale wurden Verdünnungsreihen verschiedener in Tabelle 1 zusammengefaßter Fettsäuremethylester durchgemessen. Während z. B. nach Veraschen und densitometrischer Auswertung (3) gleiche Mengen an Stearinsäure$\left(C_{18: 0}\right)$ und Linolsäuremethylester $\left(C_{18: 2}\right)$ gleiche Flächenintegrale liefern, treten bei der UV-Absorptionsmessung bei $196 \mathrm{~nm}$ entsprechend der Zahl und der Lage der Doppelbindungen erhebliche Unterschiede im Absorptionsverhalten auf. Lipide, die nur gesättigte Fettsäuren enthalten, zeigen bei $196 \mathrm{~nm}$ keine Absorption, da für die Ester-Carbonyl-Bindung $\epsilon_{\max } \cong 60$ 
beträgt gegenüber cinem Wert $\epsilon_{\max } \cong 10000$ für eine $\mathrm{C}=\mathrm{C}$ Doppelbindung.

Tab. 1. Relative Flächenintegrale verschiedener Fettsäuremethylester.

\begin{tabular}{lllc}
\hline Fettsäure & rel. Fläche & Fettsäure & rel. Fläche \\
\hline$C_{18: 0}$ & 0,0 & $C_{18: 1(n-9)}$ & 7,2 \\
$C_{11: 1(n-1)}$ & 4,0 & $C_{18: 2(n-6)}$ & 8,7 \\
$C_{16: 1(n-7)}$ & 7,0 & $C_{18: 3(n-3)}$ & 12,0 \\
\hline
\end{tabular}

Dieses für eine quantitative Bcstimmung zunächst störend erscheincnde Absorptionsverhalten läßt sich durch Wahl geeigneter Standards (natürliche Lipide) ausschalten. Bei der Bestimmung der Lipide aus Serum und Gewebe tritt dieses Absorptionsverhalten nur dann als Meßfehler in Erscheinung, wenn der Desaturierungsgrad der natürlichen Lipide stark von der Norm abweicht.

Nach den von Schrade durchgeführten gas-chromatographischen Bestimmungen der Verteilung der Fettsäuren aus Gesamtlipid (4) und den cinzelnen Lipidfraktionen des Serums (5) lassen sich nach der Formel:

$$
\overline{D B}=\frac{\sum_{i=0}^{i=6} P_{i} \cdot i}{\sum_{i=0}^{i} P_{i}+P_{r}}
$$

$\overline{\mathrm{DB}}=$ mittlere Doppelbindungszahl

$P_{i}=$ prozentualer Anteil der Fettsäuren mit i Doppelbindungen

$\mathrm{i}=$ Zahl der Doppelbindungen

$\mathrm{P}_{\mathrm{r}}=$ prozentualer Anteil der nicht identifizierten Fettsäuren

die in Tabelle 2 angegebenen mittleren Doppelbindungszahlen berechnen.

Tab. 2. Mittlere Doppelbindungszahlen einzelner Lipidfraktionen sowie der Gesamtfettșäuren für vier Personen-Kollektive.

\begin{tabular}{lllll}
\hline & \multicolumn{3}{l}{ Kollektiv Kollektiv } & \multicolumn{2}{l}{ Kollektiv } \\
& I & II & III & IV \\
\hline CHE & 1,61 & 1,54 & 1,40 & 1,48 \\
TG & 1,01 & 0,97 & 0,90 & 0,94 \\
FFS & 0,88 & 0,89 & 0,78 & 0,83 \\
PL & 1,28 & 1,27 & 1,09 & 1,18 \\
Gesamt FS-berechnet & 1,29 & 1,25 & 1,12 & 1,19 \\
Gesamt FS-gemessen & 1,00 & - & 0,83 & $0,93 *$ \\
\hline
\end{tabular}

* Bei der Aufarbeitung in die verschiedenen Fraktionen erfolgte offenbar ein Verlust an mittelkettigen gesättigten Fettsäuren.

Kollektiv I = Gesunde 16-41 Jahre

Kollektiv II = Gesunde 46-71 Jahre

Kollektiv III = Atherosklerotiker mit Hyperlipoproteinaemie

Kollektiv IV = Atherosklerotiker mit Normolipoproteinaemie

Aus den Tabellen 1 und 2 geht hervor, daß bei Kollektiv III mit der extremsten Abweichung der Doppelbindungszahlen gegenüber Kollektiv I ein Meßfehler von höchstens 5\% zu erwarten ist.

Für die in Abbildung 2 aufgeführten Lipide: Cholesterinester (Cholesterinlinolenat; Eastman); Triglyceride (Maiskeimöl); Cholesterin (biochemisch rein; Merck); Fettsäuren (GC-Referenzsubstanzen; Merck); Lecithin (Sojalecithin; Nattermann, Köln) wurden die Linearitätsbereiche bestimmt.

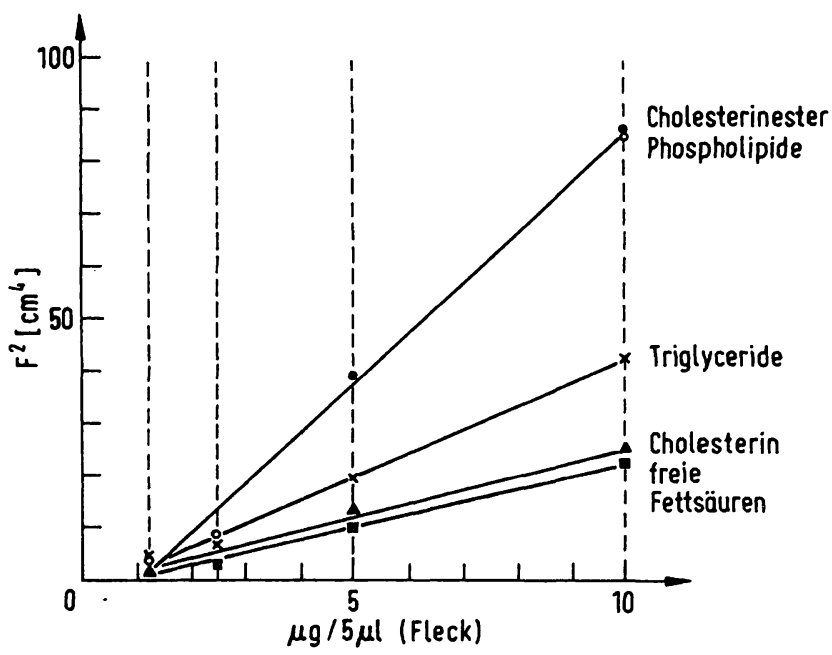

Abb. 2. Linearitätsprüfung der Flächenintegrale verschiedener Lipidklassen gegen dic aufgetragene Menge.

- Cholesterinester, $x-\times$ Triglyceride $\Delta-\Delta$ Cholesterin, $\square-\square$ freie Fettsäuren, - - Phospholipide.

Wie Abbildung 2 zeigt, sind die Flächenintcgrale im Bereich von $1 \mu \mathrm{g}-8 \mu \mathrm{g}$ linear und damit der aufgetragenen Lipidmenge direkt proportional.

Wird bei der Auswertung nur ein Teil der Substanzbande optisch erfaßt, dann ist unbedingt auf eine Konstanz der Laufstrecken zu achten, da unterschiedliche Bandenbreiten zu Meßfehlern führen. Diese Meßfehler können vermieden werden, wenn die Platten nicht in Laufrichtung, sondern quer dazu über jeweils eine Lipidklasse gemessen werden. Hierbei wird eine eventuell unter dieser Bande liegende Backgroundzone als Nullwert gemessen und somit eliminiert. Abbildung 3 zeigt, daß dann sogar eine noch bessere Linearität zwischen Flächenintegral und aufgetragener Lipidmenge besteht.

Hierbei kann für jede Lipidklasse eine eigene Ausschlagsoptimierung eingestellt und das Flächenintegral mit Standardsubstanzen verglichen werden. Auf einer DC-Platte $100 \times 100 \mathrm{~mm}$ könner 6 Seren und zwei Standardgemische aufgetragen, getrennt und gemessen werden. Als Standardsubstanzen sollten in der Fettsäureverteilung analoge Substanzen Verwendung finden.

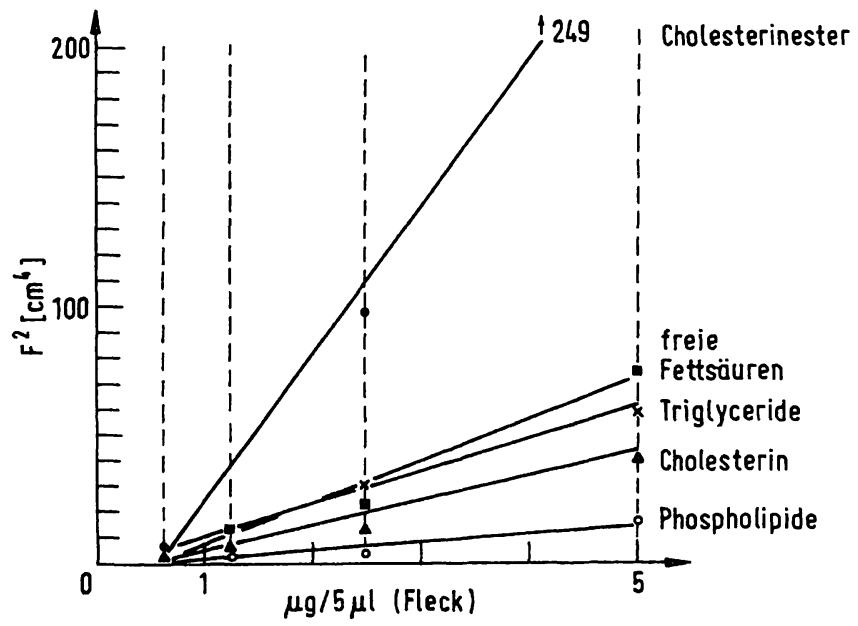

Abb. 3. Linearitätsprüfung der Flächenintegrale gegen aufgetragene Lipidmenge bei Messung quer zur Laufrichtung. - - Cholesterincster, $x-x$ Triglyceride, $\triangle \longrightarrow \Delta$ Cholesterin, $\square-\square$ freie Fettsäuren, ○-० Phospholipide 


\section{Serumlipidanalysen}

a) auf Objektträgern

Die Herstellung der Platten, die Lipidextraktion und die Gesamtlipidbestimmung erfolgt wie früher beschrieben (3). Wie aus den Abbildungen 2 und 3 hervorgeht, ist eine Linearität zwischen Flächenintegral und aufgetragener Lipidmenge zwischen $1 \mu \mathrm{g}$ und $8 \mu \mathrm{g}$ gegeben. Dieser Bereich wird bei einem Gesamtlipidgehalt von $5,00-8,00 \mathrm{~g} / 1$ Serum eingehalten, wenn man folgendermaßen verfährt:

$50 \mu \mathrm{l}$ Serum $(8,00 \mathrm{~g} / 1$ Serum Gesamtlipide) werden wie beschrieben extrahiert, zur Trockne eingedampft und in $200 \mu \mathrm{l}$ Chloroform-Methanol 2/1 (v/v) aufgenommen. Davon werden $50 \mu \mathrm{l}$ zur gravimetrischen Gesamtlipidbestimmung ausgewogen (Faktor $\mathrm{F}=8$ ) und der Rest von $150 \mu \mathrm{l}$ kann für die DC-Analyse verwendet werden. Es werden $5 \mu \mathrm{l}$ (Konstriktionspipette) aufgetragen. Bei einer angenommenen Verteilung von: $40 \% \mathrm{CHE}$, $15 \% \mathrm{TG}, 15 \% \mathrm{CH}, 3 \% \mathrm{FFS}$ und $27 \% \mathrm{PL}$ ergeben sich $4 \mu \mathrm{g} \mathrm{CHE}, 1,5 \mu \mathrm{g} \mathrm{TG}, 1,5 \mu \mathrm{g} \mathrm{CH}, 0,3 \mu \mathrm{g}$ FFS und 2,5 $\mu \mathrm{g}$ PL. Alle Lipidklassen mit Ausnahme der freien Fettsäuren befinden sich im angegebenen Linearitätsbereich. Abbildung 4 zeigt die Auswertung der dünnschichtchromatographisch getrennten Lipide eines Normalserums durch UV-Absorptionsmessung in Remission bei $196 \mathrm{~nm}$.

Zur quantitativen Auswertung werden Faktoren benötigt, die den unterschiedlichen Gehalt an Doppelbindungen verschiedener Lipidklassen berücksichtigen. Als Bezugssubstanz für die Erstellung von Korrekturfaktoren

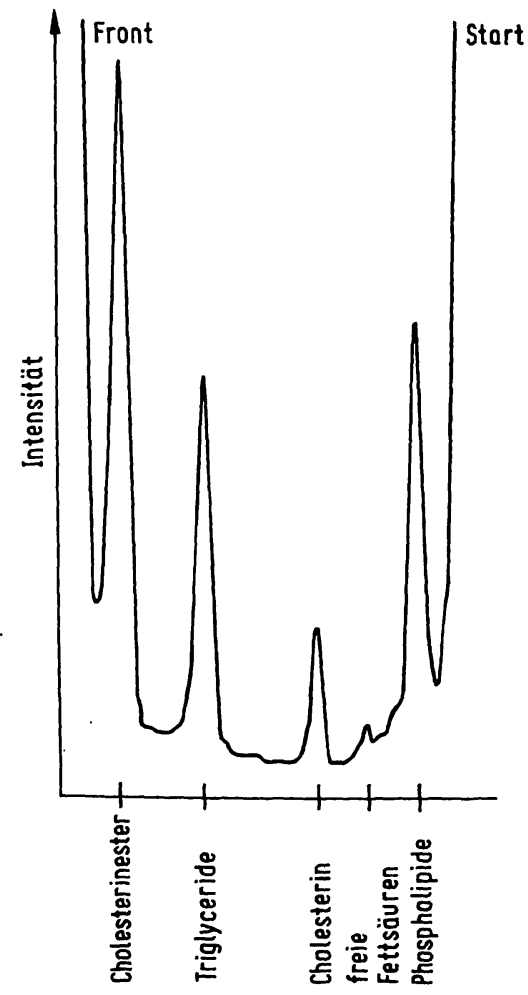

Abb. 4. UV-Remissionsmessung der Lipide eines Normalserums nach DC-Trennung auf Objektträgern. dient Cholesterin. Aus den angegebenen Doppelbindungszahlen, der Fettsäureverteilung nach Schrade $(4,5)$ und den Angaben von Gartzke et al. (2) lassen sich die in Tabelle 3 aufgeführten Korrekturfaktoren berechnen.

Tab. 3. Korrekturfaktoren für vier Lipidklassen; bezogen auf Cholesterin $=1,00$. (Die Korrekturfaktoren für vier Kollektive ergeben sich aus den für diese, Kollektive geltenden Doppelbindungszahlen).

Kollektiv I = Gesunde 16-41 Jahre

Kolkektiv II = Gesunde 46-71 Jahre

Kollektiv III = Atherosklerotiker mit Hyperlipoproteinaemie

Kollektiv IV = Atherosklerotiker mit Normolipoproteinaemie

\begin{tabular}{lllll}
\hline Lipid & $\begin{array}{l}\text { Kollektiv } \\
\text { I }\end{array}$ & $\begin{array}{l}\text { Kollektiv } \\
\text { II }\end{array}$ & $\begin{array}{l}\text { Kollektiv } \\
\text { III }\end{array}$ & $\begin{array}{l}\text { Kollektiv } \\
\text { IV }\end{array}$ \\
\hline CHE & 0,64 & 0,66 & 0,70 & 0,68 \\
TG & 0,73 & 0,76 & 0,82 & 0,79 \\
FFS & 0,80 & 0,79 & 0,91 & 0,85 \\
PL & 0,79 & 0,80 & 0,93 & 0,86 \\
\hline
\end{tabular}

Die Auswertung des in Abbildung 4 gezeigten Absorptions-Chromatogramms ergibt eine Lipidverteilung von: $39 \% \mathrm{CHE}, 27 \% \mathrm{TG}, 11 \% \mathrm{CH}, 1,2 \% \mathrm{FFS}$ und $22 \% \mathrm{PL}$.

b) auf $100 \times 100 \mathrm{~mm}$ Platten (quergemessenn)

Abbildung 5 zeigt die Absorptionsmessung von 8 CHEFlecken, quer zur Laufrichtung gemessen. Bei Verwendung von Standards lassen sich die Lipidmengen direkt in $\mathrm{g} / \mathrm{l}$ Serum angeben.

\section{Fehlerbreite und Variation}

Die Bestimmung der Standardabweichung und der Variation erfolgte nach folgenden Formeln:

Standardabweichung: $s= \pm \sqrt{\frac{\sum^{n}(\bar{x}-x)^{2}}{(n-1)}}$

Variation:

$$
\mathrm{V}=\frac{\mathrm{s} \cdot 100}{\overline{\mathrm{x}}}
$$

mit

$\mathrm{x}=$ Wert der Einzelmessung

$\overline{\mathrm{x}}=$ Mittelwert der Einzelmessungen

$\mathrm{n}=$ Zahl der Einzelmessungen

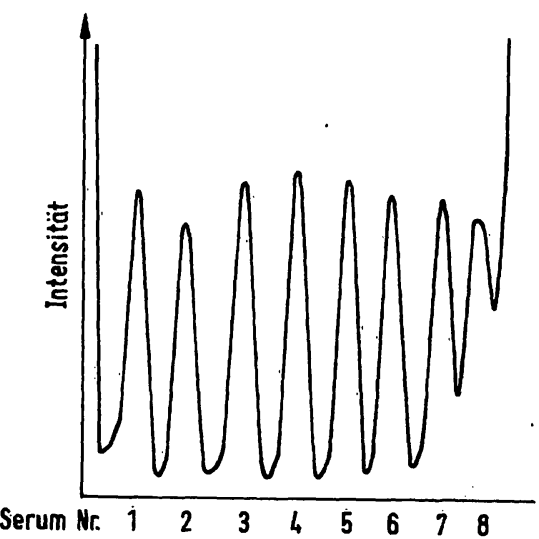

Abb. 5. UV-Remissionsmessung von 8 CHE-Flecken aus 8 Seren quer zur Laufrichtung gemessen. 
Tab. 4. Prozentuale Verteilung, Standardabweichung und prozentualer Fehler bei der Bestimmung der Lipide aus je $50 \mu 1$ Serum eines Patienten mit Hypercholesterinaemic. (In Klammern der entsprechende Wert der densitometrischen Auswertung), $n=10$.

\begin{tabular}{lrrrrr}
\hline Komponente & CHE & TG & CH & FFS & PL \\
\hline \% Gesamtlipid & $50,1(50,7)$ & $6,8(7,1)$ & $13,4(14,6)$ & $1,6(1,5)$ & $28,1(26,1)$ \\
Standardabweichung: s & $3,2(2,1)$ & $0,8(0,6)$ & $0,8(0,8)$ & $0,4(0,3)$ & $2,7(2,0)$ \\
Variation (\%) & $6,5(4,2)$ & $12,6(8,2)$ & $5,6(5,7)$ & $28,5(20,7)$ & $9,6(7,8)$ \\
\hline
\end{tabular}

Die Auswertung dünnschichtchromatographisch getrennter Lipide durch UV-Absorptionsmessung in Remission soll eine Alternativlösung zur früher beschriebenen densitometrischen Auswertung darstellen. Der Vorteil liegt im zerstörungsfreien Nachweis und einer Zeitersparnis. Ferner entfällt das Besprühen mit Schwefelsäure.

Eine Störung der Messung durch andere im UV absorbierende Bestandteile des Serums konnte bei der verwendeten Extraktionsmethode nicht beobachtet werden. Das Einschleppen von UV-aktiven Verunreinigungen, wie Phthalester über Plastikspritzen, Zentrifugenröhrchen, Lösungsmittel etc. ist peinlichst zu vermeiden.

Mit beiden beschriebenen Methoden ist es möglich, aus $50 \mu \mathrm{l}$ Serum oder $10-20 \mathrm{mg}$ Gewebe die Hauptlipidklassen quantitativ zu bestimmen.

\section{Literatur}

1. Khurt, N. H. \& Rouser, G. (ed.) (1965) $15^{\text {th }}$ A. O. C. S. Symp. on quantitative methodology in lipid research, J. Amer. Oil Chemists Soc. 42, 257-304; 353-416; 481-528; $569-616$ und $665-712$.

2. Gartzke, J. \& Nolte, K. D. (1973), J. Chromatography (Amsterdam) $84,109-115$.

\section{Danksagung}

Wir danken

Herrn Dr. Betzing, Fa. Nattermann, für die freundliche Überlassung von reinem Sojalecithin.

Herrn K. H. Kasimir für die sorgfältige technische Mitarbeit.

Herrn Dr. Rolf Schmieder von der Carl Zeiss Zweigniederlassung Köln (Carl Zeiss, Zweigniederlassung Köln, 5 Köln, Blaubach 13) für viele praktische Hinweise, Diskussionen und für die Möglichkeit, im Kölner Applikationslabor Proben durchmessen zu können.

Die Arbeit wurde durch Sachmittel der Deutschen Forschungsgemeinschaft unterstützt.

3. Egge, H., Murawski, U. Müller, J. \& Zilliken, F. (1970), diese Z. 8, 488-491.

4. Schrade, W., Böhle, E., Biegler, R., Meder, V. \& Teicke, R. (1960), Klin. Wochenschr. 38, 126-134.

5. Schrade, W., Böhle, E., Biegler, R., Teicke, R. \& Ullrich, B. (1960), Klin. Wochenschr. 38, 739-753.
Dr. U. Murawski 53 Bonn

Nußallee 11 